\title{
Contracting the Facebook API ${ }^{*}$
}

\author{
Ben Rubinger and Tevfik Bultan \\ Computer Science Department \\ University of California \\ Santa Barbara, CA 93106, USA \\ \{brubinger, bultan\}@umail.ucsb.edu
}

\begin{abstract}
In recent years, there has been an explosive growth in the popularity of online social networks such as Facebook. In a new twist, third party developers are now able to create their own web applications which plug into Facebook and work with Facebook's "social" data, enabling the entire Facebook user base of more than 400 million active users to use such applications. These client applications can contain subtle errors that can be hard to debug if they misuse the Facebook API. In this paper we present an experience report on applying Microsoft's new code contract system for the .NET framework to the Facebook API. We wrote contracts for several classes in the Facebook API wrapper which allows Microsoft .NET developers to implement Facebook applications. We evaluated the usefulness of these contracts during implementation of a new Facebook application. Our experience indicates that having code contracts provides a better and quicker software development experience.
\end{abstract}

\section{Introduction}

Recently, there has been a significant advancement in the proliferation of the social networking phenomenon. Websites such as Facebook.com allow users to connect in new ways: sharing information with one another and creating personal online "social networks" by designating other users of the site as online friends.

As of May, 2007, software developers can develop their own applications for Facebook. These applications allow software developers to provide users of their applications functionality beyond what the original Facebook website offers. Just as the original Facebook website does, these third party applications can take advantage of the social graph (the collection of friendship relationships specific to each user) to provide exciting and novel user experiences.

Examples of third party Facebook applications that currently exist include applications that allow users to share music tastes with their friends, give custom questionnaires/polls to their friends, allow their friends to leave them anonymous messages, share information about the locations where they have traveled, and more. The common theme of these applications is that they allow interaction and observation across the social axis by accessing the friendship relationships of users that are stored by Facebook.

There is a huge potential user base for third party Facebook applications. Facebook reports that they have 400 million active users, of which 50\% sign in daily. Hence, there are many software developers writing third party Facebook applications for this market. There are often several different applications performing similar functions competing for market share. For these applications to be successful, the user experience needs to be positive. Facebook has built in mechanisms which let users know what applications their friends use. Furthermore, there is a Facebook application directory where end users have the ability to give specific applications ratings and to see statistics about application usage.

*This work is supported by NSF grants CCF-0916112 and CCF-0716095.

Salaün, Fu, and Hallé (Eds.): Fourth International Workshop on Testing, Analysis and Verification of Web Software

EPTCS 35, 2010, pp. 63-74 doi 10.4204/EPTCS.35.6 (c) B. Rubinger \& T. Bultan

This work is licensed under the Creative Commons Attribution License. 
A third party Facebook application that is not dependable is unlikely to be successful. Yet it is challenging to write bug-free third party Facebook applications. Third party Facebook applications use the Facebook API to access the friendships relationships among users. An incorrect call to a Facebook method can cause a fatal error for any third party application. Hence, a crucial factor in writing dependable third party Facebook applications is the correct usage of the Facebook API. However, the behavior of Facebook API methods are mainly described in English in the comments provided in the Facebook API. Such informal specifications can be misunderstood and can lead to errors. Moreover, these errors can be hard to find and debug since there is no automated way of tracking such violations.

In this paper we investigate application of the code contracts to the Facebook API to improve the dependability of third party Facebook applications. Code contracts allow developers to specify method preconditions, postconditions and class invariants in a way that is verifiable at runtime and in some cases statically at compile time.

Design by contract has been an influential concept in software engineering in the last two decades [6]. Many tools have been developed for analyzing contracts. Some of these tools use the host language expressions to write contract clauses and support runtime monitoring of contracts [5]. Some tools require the use of a separate annotation language such as Java Modeling Language (JML) or Spec\# for writing contracts [2, 1]. In addition to runtime monitoring, there has also been significant amount of work on static verification of contracts [4]. There has also been work on automatically inferring contracts by observing executions of a system [7], although a combination of automatically inferred contracts and hand written contracts seems to be the most effective approach [8].

Code contracts can be especially useful for third party application developers. They can serve as a contract between the organization that provides the API and the third party developers that use it. Code contracts can be used to specify the conditions that a third party application has to establish on the arguments that are passed to an API method (i.e., pre-conditions) and the conditions that the API method is expected to establish (i.e., post-conditions). Expressing these conditions using code contracts has many advantages:

- The API contract would provide an unambiguous specification of how to use the API instead of the potentially ambiguous descriptions provided in comments.

- Using contract monitoring tools the API contract conditions can be tracked at runtime. This would provide a useful testing tool for finding bugs in third party applications.

- Using static analysis tools, some classes of contract violations can be detected statically, eliminating bugs early before runtime.

- The API contract would help in debugging third party applications by identifying causes of the bugs and where to assign the blame in case of a contract violation.

In this paper we present an experience report on applying code contracts to the Facebook API. In particular we used the code contract system provided by the Visual Studio 2010 (the integrated development environment for doing .NET development) and wrote contracts for the Facebook API using this code contract system. We have focused on several classes in the Facebook API that provides access to social data stored by Facebook. We also conducted a case study on a Facebook application to evaluate the effectiveness of code contracts in third party Facebook application development. The Facebook application we developed is called Pacebook and it enables users to log their runs, review their prior runs and share them with their friends. Our experience in using code contracts in development of Pacebook and for the Facebook API suggests that code contracts are useful for third party application developers since they help both in identifying bugs and removing them quickly. 
The rest of the paper is organized as follows: In Section 2 we described the Facebook application that we used as a case study. In Section 3 we give an overview of the Facebook API and in Section 4 we give an overview of the Microsoft code contract system that we used. In Section 5 we describe how we applied code contracts to the Facebook API and in Section 6 we discuss our results. Finally, in Section 7 we conclude the paper.

\section{A Facebook Application for Run Logging}

Pacebook is a third party Facebook application that allows Facebook users to keep track of their runs/jogs by logging them and by reviewing past entries. In addition, users are able to view information about their Facebook "friends." Users can log details such as the date of the run, the distance of the run and the duration of the run. Friends (both Pacebook users and other Facebook users) are able to then post comments about runs that their friends post. Users are also able to record other friends of theirs who joined them on their runs. Facebook refers to this process of marking others who are in content as "tagging." (Often this is done to mark individuals who appear in photos.) If another user who was tagged in a run is not currently a user of Pacebook, the logging still takes place on the user's behalf.

When the user logs into Facebook, a link to the Pacebook application appears on the right-hand side of the screen. Clicking this application link launches PaceBook in the context of Facebook, providing an integrated social experience. The screenshot in Figure 1 shows the interface for logging a run. (Note that in the following screenshots, last names have been redacted for privacy reasons.)

There are several noteworthy points about the screenshot shown in Figure 1

- The address of the application: The address being pointed to in the address bar points to the facebook.com domain (http://apps.facebook.com) even though this is a third party application. This is interesting because the third party application is hosted on a separate web server, not on Facebook's servers. After an initial request is made to the Facebook site, another request is made to the third party web server to pull the application content.

- The chat content on the right hand side: Facebook provides a chat facility using CSS styling to simulate a chat window which is prevalent throughout the entire Facebook session, including while using Pacebook. Users can engage in real-time online chat during their Pacebook experience if they choose.

- The friend selector toward the bottom of the screenshot: This is achieved with the Facebook multifriend input. This allows the user to tag his or her friends in the run. The names with which it is populated come from the user's Facebook friend list, and it features an autocomplete feature which uses AJAX to dynamically populate suggestions as the user is typing. This provides a user experience that is consistent with other Facebook features.

The screenshot in Figure 2 shows a new entry in first author's newsfeed which was automatically created by Pacebook announcing to all Facebook friends of the first author of his recent run. Notice that underneath the status update for Ben Rubinger, you see the text "via PaceBook." This is an application link allowing others to get info on Pacebook, and ultimately add the application to their online profile so it shows up in the left-hand navigation and they can begin using it. The Facebook users who see the entry generated by the Pacebook can interact with it for example by "liking" it (placing a thumbs-up sign on it) or by commenting on it. 


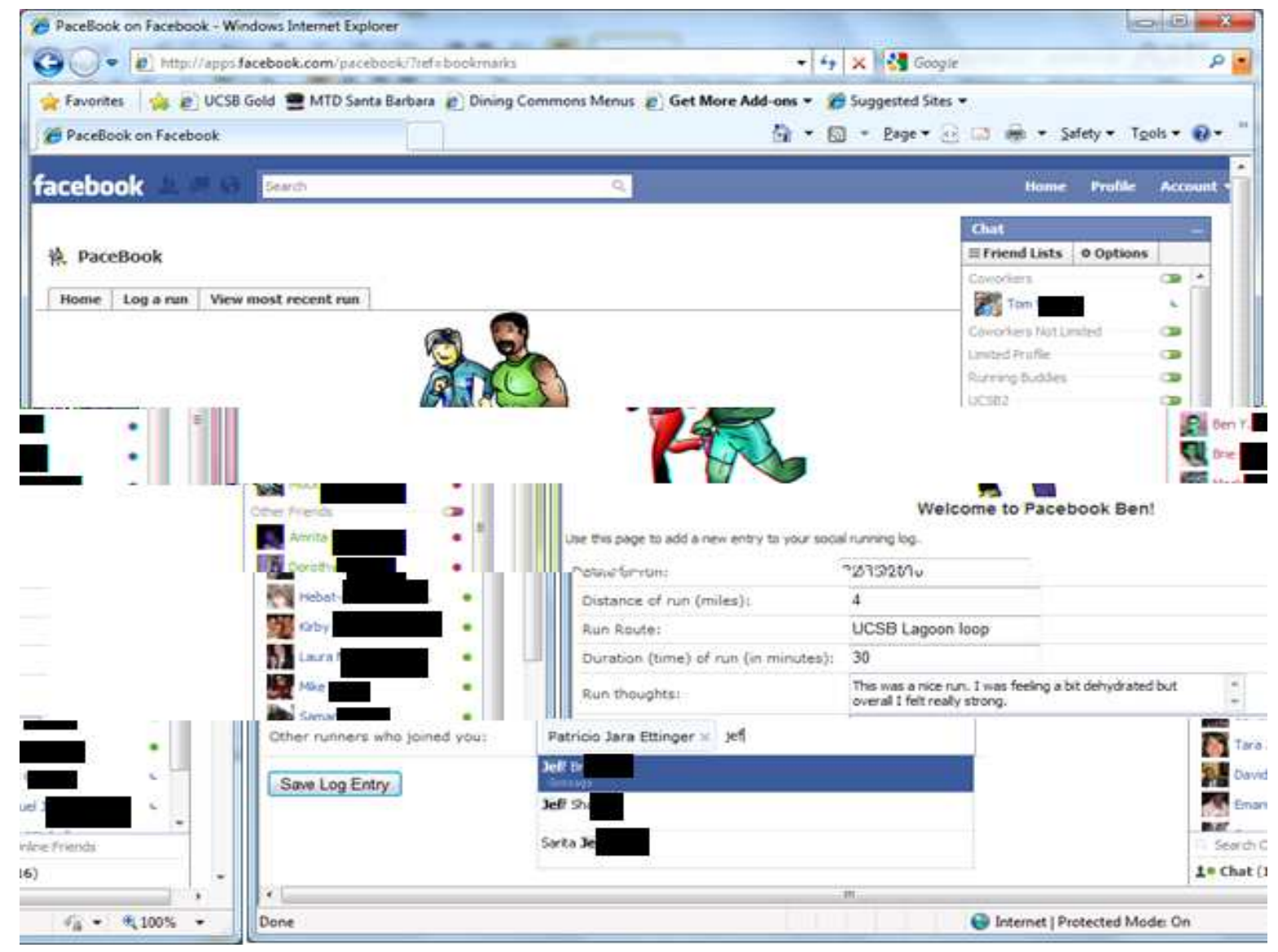

Figure 1: Pacebook: A Facebook Application

\section{Facebook API Overview}

The way that a developer interacts with the Facebook system is through the Facebook API. This API contains methods that allow developers to interact with the application user's social data. Examples of methods in the API are:

- User.GetFriends() This returns a list of the application user's Facebook friends. Applications might make use of this data to give the user a list of his or her friends to choose with whom to interact in the context of a third party application. In the context of Pacebook, this function is useful to allow the user to "tag" other friends that were on the run.

- Data.GetObject(long objectid) This method allows objects stored in the Facebook data store (a data store created for Facebook third party developers to persist application data) to be retrieved.

To create a Facebook application, several different language options are available, each having different implications. The development client library API provided directly by Facebook company is the PHP 5 client library. As such, applications written for this library are written in PHP and would behave as any application running in the context of this scripting language. 


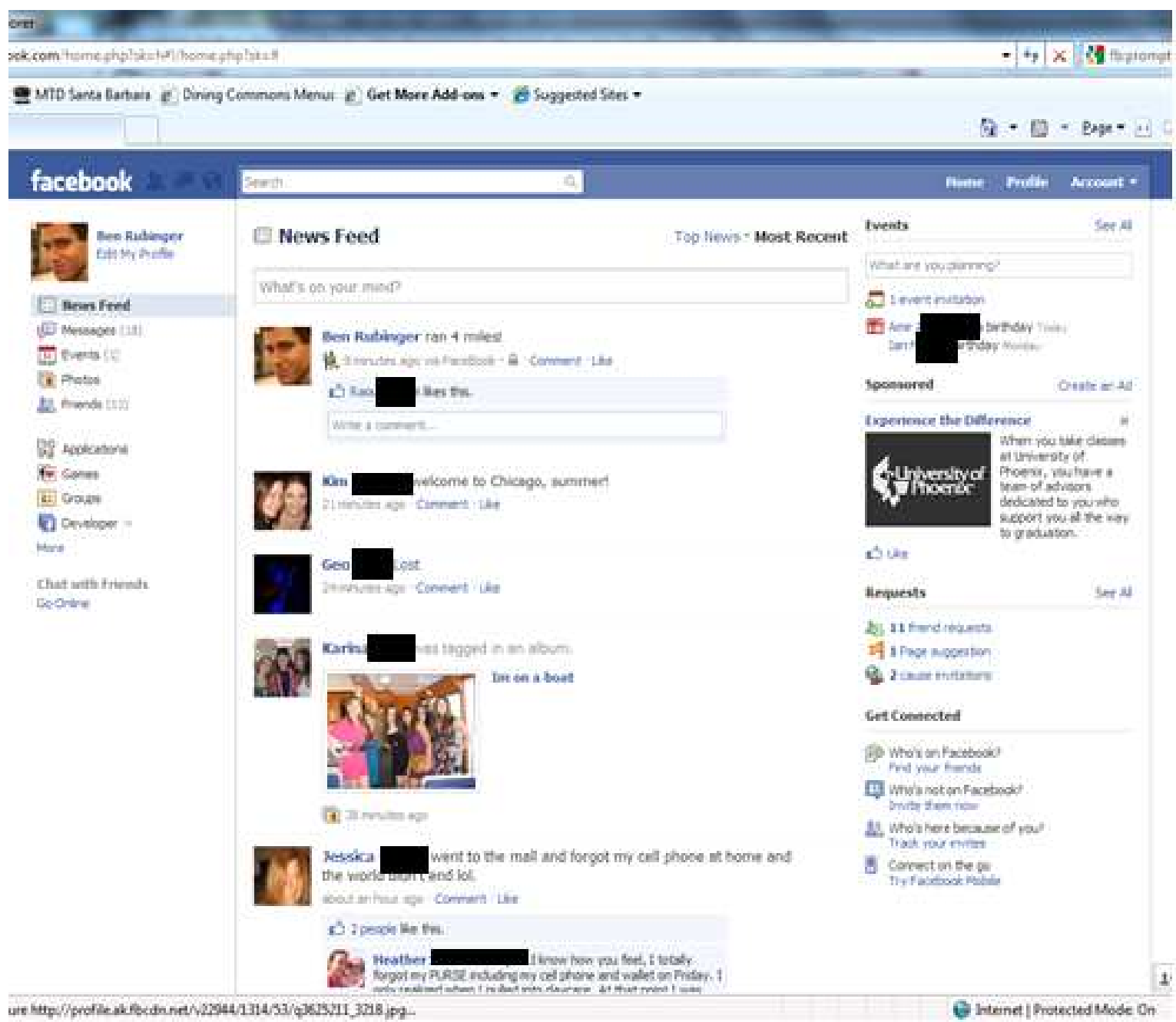

Figure 2: Pacebook in newsfeed

There are also other options available beyond PHP for Facebook development. One such example, the Microsoft SDK for the Facebook Platform allows applications written in a Microsoft .NET managed language to integrate with Facebook. These applications can be written in any managed .NET language, though typically the language used is either C\# or VB.NET. For Pacebook, C\# was used.

Throughout the rest of this paper, we will refer to the Microsoft SDK for the Facebook Platform (also known as the Facebook Developer Toolkit [3] ) as the .NET Facebook API wrapper. The .NET Facebook API wrapper is not created by Facebook; rather a third party consulting company called Clarity Consulting created this wrapper in order to allow Microsoft .NET developers to write Facebook applications. In this work we focus on writing contracts for the .NET Facebook API wrapper. The Facebook application we use as a case study is also written using the Microsoft .NET framework. In our case study we focused on the Facebook REST API in the .NET Facebook API wrapper which contains the main functionality for accessing the social data provided by Facebook. 


\section{Microsoft Code Contracts}

The Visual Studio 2010 toolset (the integrated development environment for doing .NET development) includes new tools for performing various analyses. These include support and tooling for using code contracts, as well as mechanisms to perform checking against these contracts. The contracts are specified as pre-conditions, post-conditions, and class invariants. The Visual Studio 2010 toolset includes support for runtime contract checking which can be used to improve testing. The toolset also provides support for static contract verification, and documentation generation. Not all contracts can be checked statically, but when they can, static identification of contract violations reduces the cost of testing, runtime checking and debugging.

Here is an example in the context of a simple Facebook application:

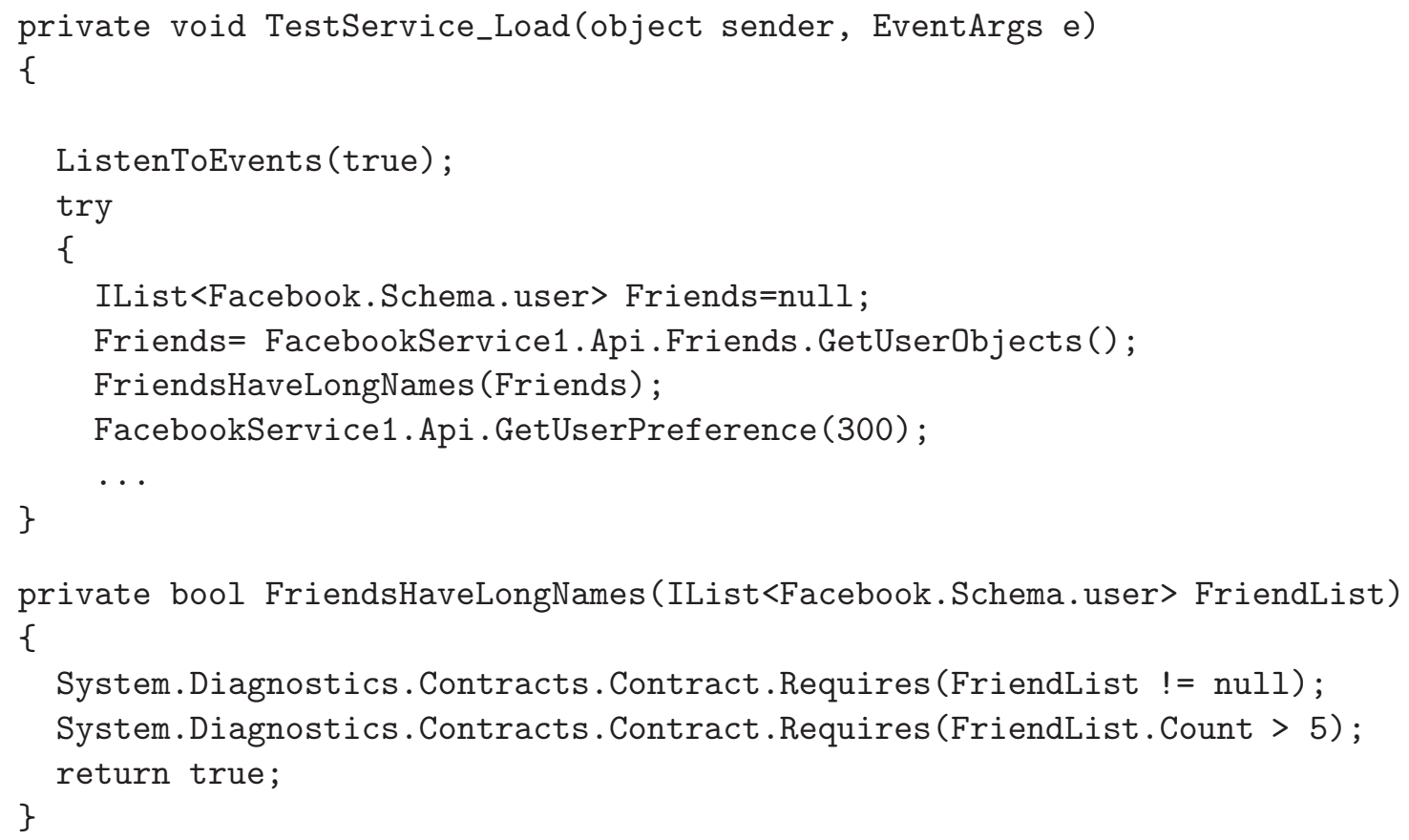

In the above $\mathrm{C} \#$ code, the method TestService_Load calls the method FriendsHaveLongNames. FriendsHaveLongNames has two method preconditions. Both preconditions (listed in "requires" calls) check a property of a "social" object whose value can not be determined statically and therefore neither can the contracts. The FriendList object passed into FriendsHaveLongNames is populated at runtime with social data (in this case, data pertaining to the current application user's friend list). The system cannot make a determination about this precondition statically. These checks will still operate dynamically (at runtime). Should the check fail at runtime, an exception is typically thrown (the runtime failed contract behavior is configurable by the developer).

Separately, making the subsequent GetUserPreference (300) call causes one of the contracts inside that method to fail statically as the constant integer value can be used to check the contract statically. See the code taken from the Facebook .NET API wrapper including the contract we have added below:

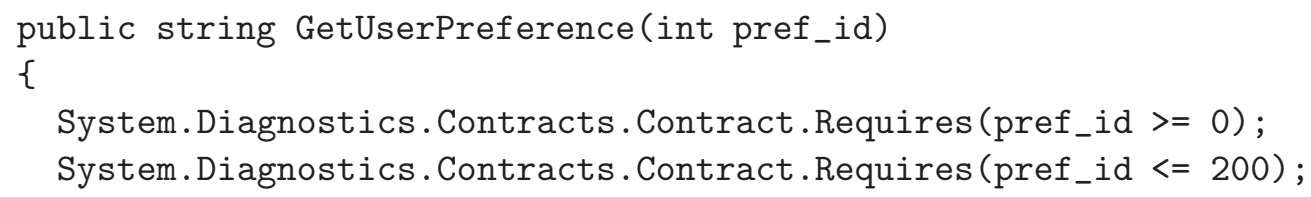




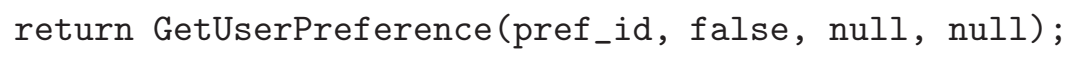

\section{Applying Code Contracts to the .NET Facebook API Wrapper}

The .NET Facebook API wrapper source is publicly available [3] which allowed us to instrument the code with contracts. The .NET Facebook API wrapper consists of many classes, we focused on a small core subset that was used by the Pacebook application. The contracts clauses were written either as a direct translation of the comments in the API documentation or they were additional checks added by us based on the API documentation interpretation.

\subsection{Contract Clauses: Translation from the Wrapper Documentation}

In many circumstances, the .NET Facebook API wrapper contains method documentation explaining, in addition to other things, details about the parameters being passed to a function. This information can be transformed into method preconditions which specify the same information as the documentation but do so in a way that the system is able to verify, providing a type of enhanced and unambiguous documentation.

The following is an example of a function which is in the .NET Facebook API wrapper source and shows the conversion of the textual documentation into a method precondition using the "Requires" contract element:

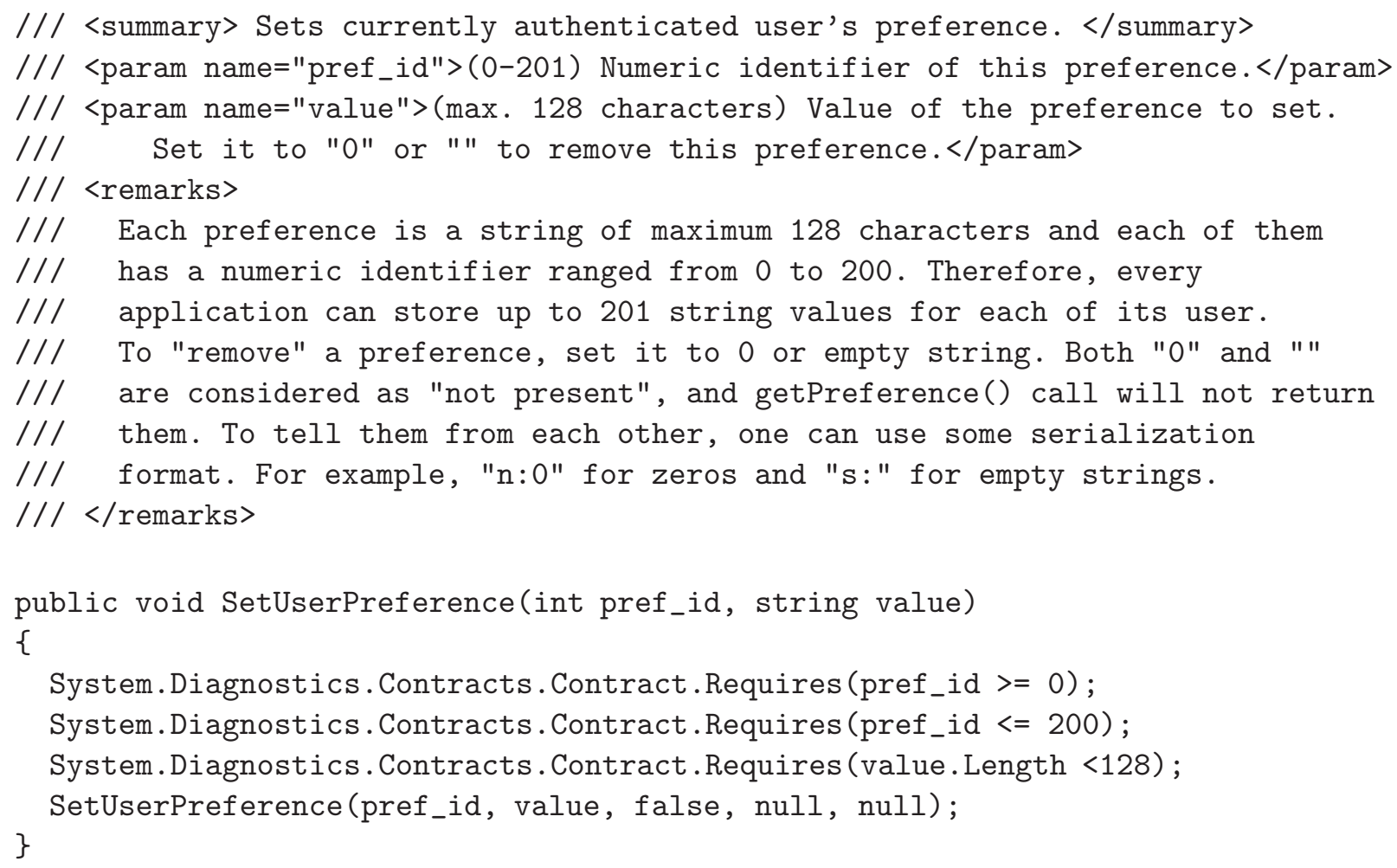




\subsection{Contract Clauses: Additional Checks}

In several circumstances, we were able to insert contract clauses beyond doing literal translation from documentation. We found that there were instances where the documentation did not specify parameter requirements that should have been specified. Below is an example from the Facebook wrapper which shows a null check added as a contract clause, while there is no mention of a non-null requirement in the documentation:

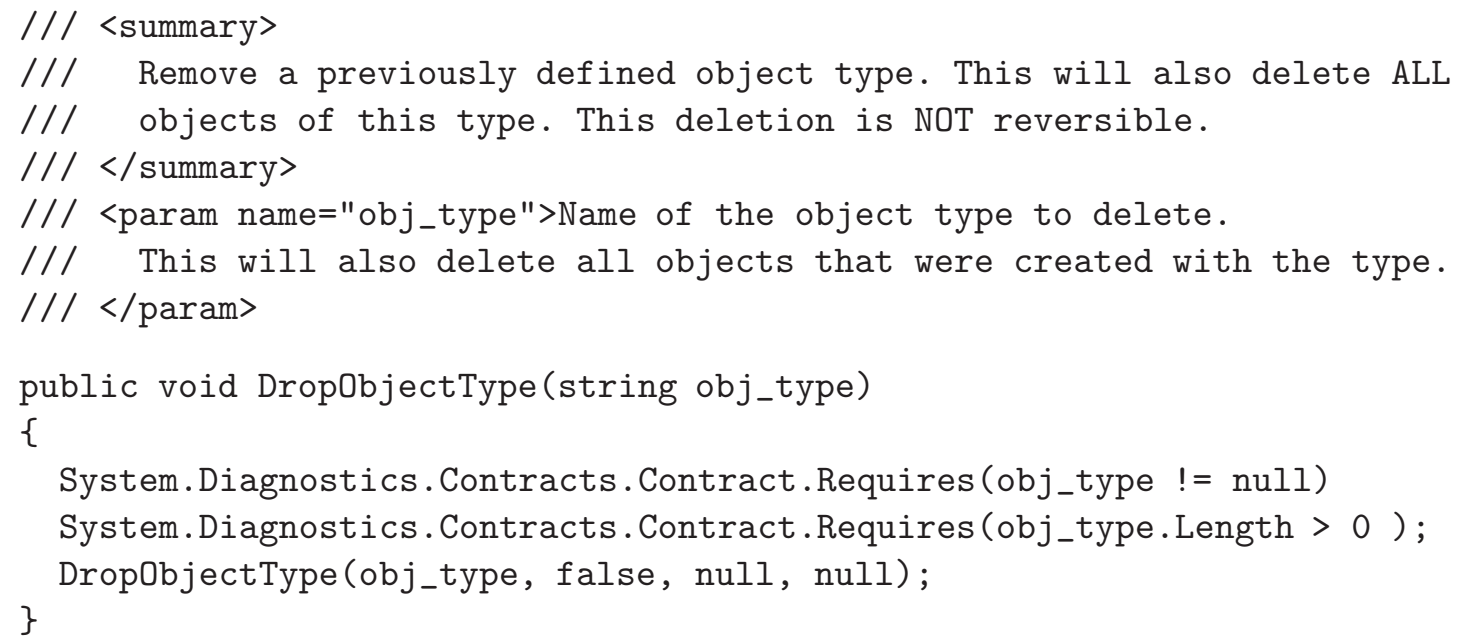

\subsection{Contract Clauses: Categories}

The contract clauses we have created for the .NET Facebook API wrapper can be divided into three categories:

1. Null checks: These ensure that a null value is not passed into a specific parameter which expects a non-null object or value.

2. Range checks: These are used to ensure that a specified parameter falls within a specified range. For example, there is a function, DefineAssociation, which creates a data association between different data types in the Facebook Data store. It has an integer parameter which specifies the type of association (1-way, 2-way symmetric and 2 way asymmetric). It only accepts values between 1 and 3 . The contract clause created to verify this constraint falls under the category of range checks.

3. Size checks: These are used to verify that the length of an object (often the length of a string or a list) meets size constraints.

\subsection{Contracts for the .NET Facebook API wrapper}

The source code for the .NET Facebook API wrapper is responsible for abstracting the details of the communication with the Facebook API. It allows the developer to write code in a managed, strongly typed .NET language like C\#, making calls which will get wrapped and sent over the wire to Facebook's servers in XML format. It also parses the responses sent via XML format from the Facebook server and populates strongly typed objects for the developer to work with.

For the .NET Facebook API wrapper, there are 32 source files in the REST API, each containing different subsections of the API wrapper. The source files we focused on were: 
- Data.cs (937 lines of code, 1403 lines of comments) which contains wrapper code for interacting with the Facebook Data store (a simple, highly scalable data store made available to Facebook third party application developers).

- Friends.cs (292 lines of code, 574 lines of comments) which contains wrapper code for getting information about "friends" in a user's social network.

- Comments.cs (127 lines of code, 237 lines of comments) which contains methods for working with comments users can post about news items that show up in the "news feed" (a list of recent friend activity displayed when the user first signs into Facebook).

- Events.cs (243 lines of code, 517 lines of comments) which contains methods for working with Facebook events, a type of calendar entry used for group scheduling.

Note that these files only contain wrapper classes that invoke the appropriate Facebook function for each request. They do not contain too many lines of code since they do not contain the implementation of the functionality.

The majority of the contract clauses we inserted to the .NET Facebook API wrapper methods use the requires construct, i.e., the majority of the checks we inserted were pre-condition checks. This is the most useful check for third party application developers since establishing the pre-condition is the responsibility of the client and a pre-condition violation would indicate an error in the third party application. In contrast, a post-condition violation would indicate an error in the Facebook implementation of the API.

The Micrososft code contract system we used also includes support for class invariants; however we have not used them in the contract we wrote for the .NET Facebook API wrapper source code. This is because as an API, .NET Facebook API wrapper does not afford much opportunity for making use of class invariants since there are not many fields of relevance (because the API maintains minimal state).

We also wrote contracts for the Pacebook application source code. Majority of the contracts for the Pacebook application ended up in the data access layer code, which interfaces between the user interface and the Facebook data store. In the contracts inserted into the Pacebook application (rather than the .NET Facebook API wrapper source), we made use of both class invariants in addition to pre- and postconditions.

The .NET Facebook API wrapper contains some public methods which in turn call private methods which include a longer method signature. For example:

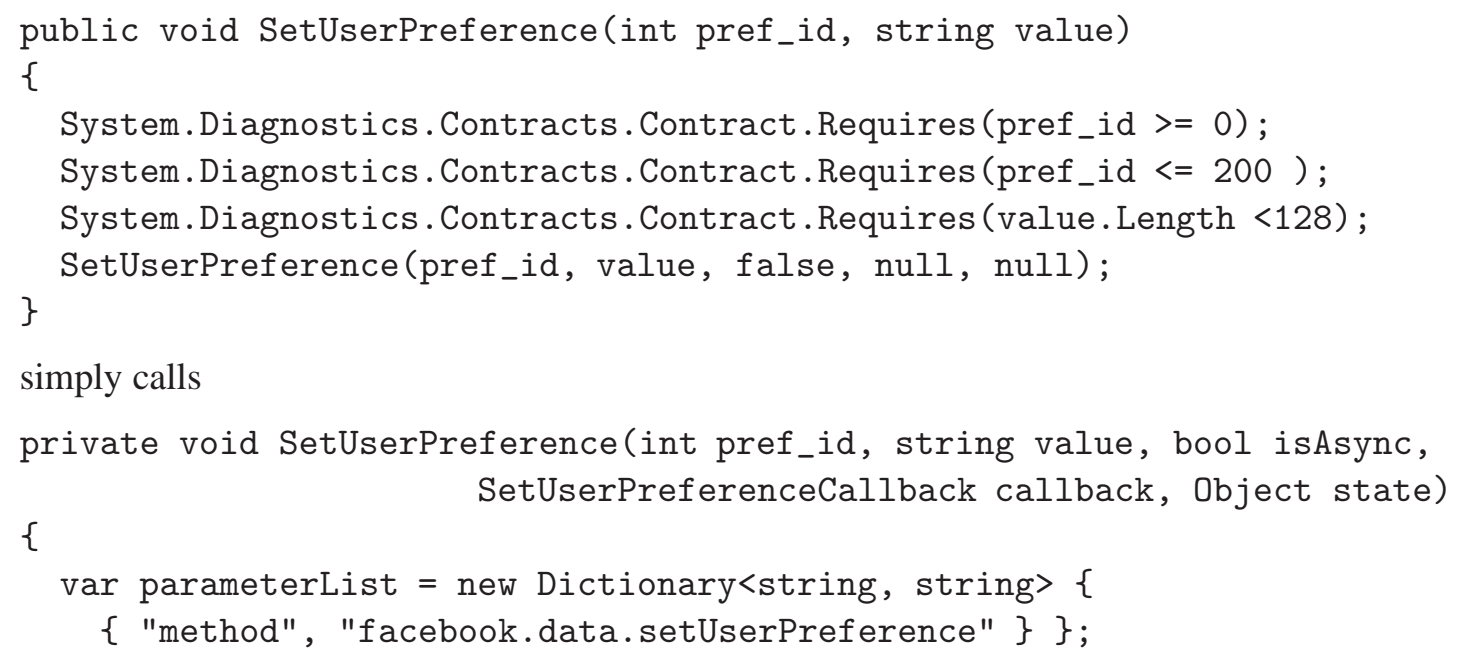




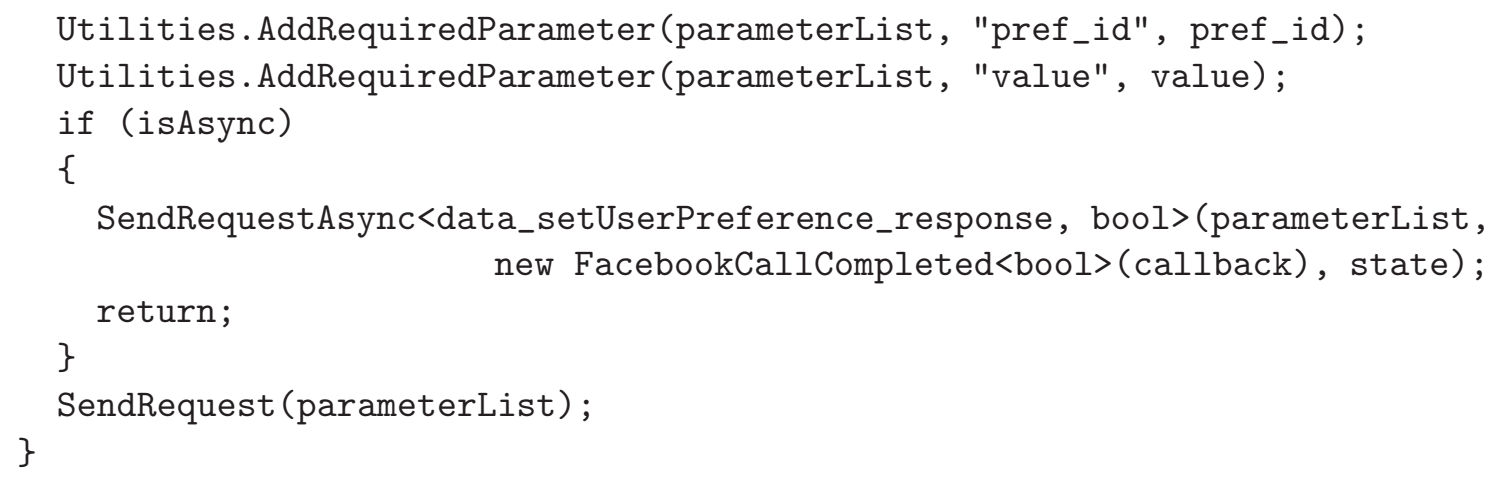

Since the developer interacts with the public method, that is where we placed the contracts as this avoids redundant checks.

The .NET Facebook API wrapper also includes asynchronous versions of some methods where the method call returns immediately without finishing the task. The task is executed on a separate thread and the caller is notified of completion by an event. This style of asynchronous interaction avoids blocking of the calling thread. We have not written contracts for this type of asynchronous methods in the API since they were not used in the Pacebook application.

\begin{tabular}{|l||c|c|c|c||c|}
\hline & Data.cs & Friend.cs & Comments.cs & Events.cs & Total \\
\hline \hline \# Methods & 39 & 10 & 6 & 11 & 66 \\
\hline \# Contracted & 33 & 8 & 6 & 9 & 56 \\
\hline Percentage & $92 \%$ & $80 \%$ & $100 \%$ & $82 \%$ & $85 \%$ \\
\hline
\end{tabular}

Table 1: Number of methods (and contracted methods) in Facebook API classes we investigated

\begin{tabular}{|l||c|c|c|c||c|c|c|}
\hline & Data.cs & Friend.cs & Comments.cs & Events.cs & Total & Contracted & Percentage \\
\hline \hline \# Parameters & 92 & 17 & 17 & 21 & 147 & 101 & $69 \%$ \\
\hline \# Nullable & 60 & 7 & 11 & 16 & 94 & 76 & $81 \%$ \\
\hline \# Range-able & 79 & 11 & 17 & 7 & 114 & 32 & $28 \%$ \\
\hline \# Size-able & 55 & 4 & 11 & 5 & 75 & 26 & $35 \%$ \\
\hline
\end{tabular}

Table 2: Number of method parameters (and contracted method parameters) in Facebook API classes we investigated

Tables 1 and 2 provide some statistics about the contracts we wrote for the Facebook API. Table 1 gives the number of methods in the four files of the Facebook API that we have investigated. For each of them, we also specify the number of methods that we wrote contracts for. Overall we wrote contracts for $85 \%$ of the methods. For the rest of the methods there were no conditions described in the API documentation and we were not able to find interesting contract clauses to write.

In Table 2 we provide data about the number of parameters for the methods we investigated in the Facebook API. We categorized the parameters as parameters that can be constrained with null (nullable), range (range-able) or size (size-able) constraints respectively. Note that these categories are not mutually exclusive. For example a list can have both a size constraint on it identifying a maximum size and a null constraint which may state that it should not be null. In Table 2 we also identify the percentages of each type of parameters that were constrained with a contract clause. Not surprisingly, the most 
common contract clauses were on null values. The percentages for range-able and size-able parameters are relatively low but this should be expected since, for example, although any integer parameter can be range-able most of them may not have a specified range in the API specification. As seen in the first row of Table 2 overall, $69 \%$ of the parameters had some type of contract clause written about them, restricting their possible values.

\section{Discussion}

The process for searching for bugs in the code that interacts with the Facebook API is often cumbersome. Code is created or modified by the developer, and then to test this code, it is deployed to the web server, and then the web site must be accessed via a web browser to execute it within the context of the server through the Facebook website. This is necessary because the code will be calling the Facebook API and this additional runtime dependency adds complexity to the debugging process. The developer then can see if there is an unexpected runtime behavior such as an exception being thrown to identify the bugs.

Let us contrast this experience with the new experience we achieve with the modified source code which includes the code contracts. For example, if we look at the method GetUserPreference, we can see that the API has a parameter for id of the stored preference. This parameter can only accept the values from 0 to 200. If a developer were to be unaware of this restriction or make a mistake and use an invalid value, to discover this error, he or she would go through the following process:

- Upload the new code to the web server

- Attempt to view the corresponding web site

- View the relevant exception which was thrown.

This is a time consuming and cumbersome multistep process. Conversely, with the static contract checking features in the Microsoft code contracts system paired with the enhanced contracts-added version of the .NET Facebook API wrapper source code, the developer is prompted that she/he made a coding error after typing in the wrong value for the input parameter and the violated contract clause is highlighted to inform the developer. This process is of course dependent on the system's ability to statically determine the value that will be used for the specific parameter. Even if the value cannot be determined statically, during runtime the contract system will inform the developer about any contract violation and identify the contract clauses that are violated, which significantly improves the efficiency of the debugging process.

In order to track the usefulness of the contracts we inserted, throughout the development of the Pacebook application we tracked the number of defects that were caught by the contract system. There were eight defects that were caught with the aid of the inserted code contracts. Of those defects, six were caught by contracts in the .NET Facebook API wrapper source, while the other two were caught by contracts in the Pacebook application source. Of the 6 defects caught by the contracts in the .NET Facebook API wrapper source 4 were null check violations, 1 was a range check violation and 1 was a size check violation. The defects caught by the contracts in the Pacebook application were both null check violations.

It is important to give some context regarding the above numbers. The code contracts were inserted into the source in an early stage of the development of the Pacebook application. Accordingly, the developer for the Pacebook (the first author) had reviewed the documentation, and had essentially retyped the documentation in contract form which made him familiar with the documentation and the meaning of the newly inserted contracts. Having this knowledge made the developer less likely to make some of the errors that otherwise would have been more likely. For others who develop their Facebook applications 
with the newly instrumented .NET Facebook API wrapper, it is reasonable to expect even greater value from the contracts since they will not have the same recent familiarity with the inserted contracts.

Let us now focus on a specific bug that was caught with the aid of the contracts. A call in the Pacebook application was unintentionally passing in an invalid value for a parameter of the method CreateAssociation. With the contracts plus static checking, this was caught before deployment without a need to go through the cumbersome bug finding process described above.

Another interesting bug that was discovered by contract checking was a bug in the contracts themselves. The static checker showed that a contract clause had failed for code that was not being called by the Pacebook application, but rather was being called by the API itself. It was then realized that a null check was inserted where a null parameter was acceptable. Note that this types of bugs are also very valuable to find since they show the inconsistency between the API description and its implementation. In contrast, a similar erroneous statement written as a comment as part of the API documentation cannot be discovered by contract checking and can lead to misunderstanding of the API by the third party developers. Precisely specifying the assumptions about the API using contracts enables us to check the consistency between the API implementation and its specification using contract checking.

\section{Conclusions}

Our experience shows that code contracts are a valuable tool for the development of third party Facebook applications. The contracts we have written for the Facebook API clarify the assumptions about how to interact with the Facebook API and, hence, are likely to reduce the number of errors in third party applications. We have observed that static contract checking is a useful tool for identifying the errors at compile time instead of the cumbersome manual testing process that requires the developer to launch the application. We believe that the contract we started for the Facebook API can be extended to the full API and can serve as a precise documentation of the API for the third party developers.

\section{References}

[1] Michael Barnett, Robert DeLine, Manuel Fähndrich, Bart Jacobs, K. Rustan M. Leino, Wolfram Schulte \& Herman Venter (2005): The Spec\# Programming System: Challenges and Directions. In: First Conference on Verified Software: Theories, Tools, Experiments, pp. 144-152.

[2] Lilian Burdy, Yoonsik Cheon, David R. Cok, Michael D. Ernst, Joseph R. Kiniry, Gary T. Leavens, K. Rus$\tan$ M. Leino \& Erik Poll (2005): An overview of JML tools and applications. International Journal on Software Tools for Technology Transfer 7(3), pp. 212-232.

[3] Facebook Developer Toolkit. http://facebooktoolkit.codeplex.com.

[4] Cormac Flanagan, K. Rustan M. Leino, Mark Lillibridge, Greg Nelson, James B. Saxe \& Raymie Stata (2002): Extended Static Checking for Java. In: PLDI, pp. 234-245.

[5] Murat Karaorman \& Parker Abercrombie (2005): jContractor: Introducing Design-by-Contract to Java Using Reflective Bytecode Instrumentation. Formal Methods in System Design 27(3), pp. 275-312.

[6] Bertrand Meyer (1992): Applying "Design by Contract". IEEE Computer 25(10), pp. 40-51.

[7] Jeremy W. Nimmer \& Michael D. Ernst (2002): Automatic generation of program specifications. In: Proceedings of the International Symposium on Software Testing and Analysis (ISSTA), pp. 229-239.

[8] Nadia Polikarpova, Ilinca Ciupa \& Bertrand Meyer (2009): A comparative study of programmer-written and automatically inferred contracts. In: Proceedings of the International Symposium on Software Testing and Analysis (ISSTA), pp. 93-104. 